\title{
ERRATUM to
}

S.GÄHLR: Contribution to the Theory of Generalized Derivatives, $Z$. Anal. Anw. 5 (1986), $367-376$.

On page 368 and 369 some of the symbols $D,<,=$ which are printed normal according to the 'manuscript had to be set in another way. The text is' correct as follows:

11. Now let $S$ be an ordered inner product space and $(\cdot, \cdot)$ be -its inner product. (The fact that the symbol $(\cdot, \cdot)$ is also used to denote the open interval cannot lead to any confusion.) Let us write

$$
f(q)\left\{\begin{array}{l}
>_{K} \\
={ }_{K} \\
<_{K}
\end{array}\right\} 0 \text { if }(f(q), K)\left\{\begin{array}{l}
\subseteq[0, \infty), \quad \text { but } \neq\{0\} \\
=\{0\} \\
\subseteq(-\infty, 0], \cdot \text { but } \neq\{0\}
\end{array}\right.
$$

wheré $(f(q), K)=\{(f(q), k) / k \in K\}$. Let $f(q)={ }_{K}^{\prime} 0$ if there exists a neighbourhood $U$ of the point 0 in $R$ with $q+U \subseteq Q$ and $(f[q+U], K)=\{0\}$ where $(f[q-U], K)$ $=\{(s, k) / s \in f[q+U], k \in K\}$. Moreover let $f(q)>_{K}^{\prime} 0\left(<_{K}^{\prime} 0\right)$. if not $f(q)={ }_{K}^{\prime} 0$, - but if there exists a neighbourhood $U$ of the point 0 in $R$ with $q+U \subseteq Q$ and $(f[q+U], K) \subseteq[0,+\infty)((-\infty, 0])$. For arbitrary $g: Q \rightarrow S$ let

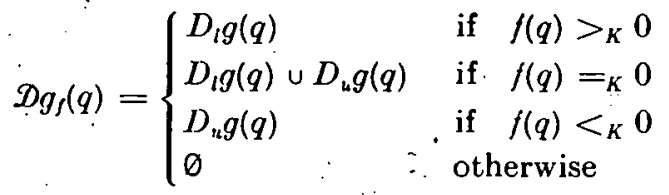

$$
\begin{aligned}
& \mathcal{D}^{\prime} f_{g}(q)=\left\{\begin{array}{ll}
D_{l} f(q) & \text { if } g(q)>{ }_{K}^{\prime} 0 \\
D_{!} f(q) \cup D_{u} f(q) & \text { if } g(q)={ }_{K}^{\prime} 0 \\
D_{u} f(q) & \text { if } g(q)<{ }_{K}^{\prime} 0 \\
\emptyset \cdot, & \text { otherwise. }
\end{array} .\right.
\end{aligned}
$$

Theorem.1: If $g$ is continuous at $q$, then.

$$
\left(f(q), \mathscr{D} g_{j}(q)\right)+\left(\mathcal{D}^{\prime} f_{g}(q), g(q)\right) \subseteq D_{l}(f, g)(q)
$$

Proof: Assume $D g_{f}(q)$ and $\mathcal{D}^{\prime} f_{g}(q)$ are not empty. Let $f(q)>_{K} 0$ and $g(q)>_{K}^{\prime} 0$ and for arbitrary $\gamma \in D_{1} g(q)$ and $\phi \in D, f(q)$ let $\psi=(f(q), \gamma)+(\phi, g(q))$. Then

Hence we have $\psi \in D_{l}(f, g)(q)$ which proves the assertion of the theorem in the case $f(q)>_{K} 0$ and $g(q)>_{K}^{\prime} 0$. The other cases can be proved analogously 1 